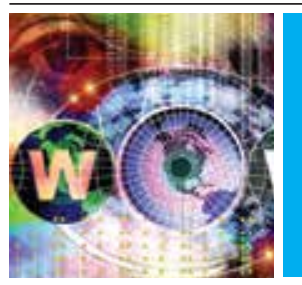

Research Article

\title{
Reputation Based Trust Management System for Improving Public Health Care System in Pakistan
}

\author{
M. Taseer Suleman, M. Hassaan Rafiq, Rafaqat Alam and M. Arsalan Tariq \\ taseersuleman@lgu.edu.pk, hassaan.rafiq@lgu.edu.pk,rafaqatalam@lgu.edu.pk, \\ arsalan.tariq@1gu.edu.pk
}

Department of Computer Science, Lahore Garrison University, Lahore, Pakistan.

\begin{abstract}
:
Health is the most important facility for the people by the government if it is provided properly. There is a lack of trust among people about healthcare system particularly in public hospitals of Pakistan. People do not trust doctors and hospitals due to poor management and low standards check-up by the doctors. This is due to the lack of feedback system which leads to the mismanagement of healthcare institutions. In this research, we have developed a reputation-based feedback management system that will overcome all the problems related to the trust of the patients regarding their medical care. This will enhance the system and will let doctors, staff, and management to work honestly to make their repute well.
\end{abstract}

Keywords: Reputation, Trust, Medical Care, Health

\section{Introduction}

Health is wealth, is common and the most known phrase in the world. Health carries most marks when it comes grading the life. If we do not have good health we carry nothing but loss. Therefore, it is indeed the best thing one can have in shape of good life. Good health eventually contributes towards the progress of country as people with sound health mentally and physically will be productive and beneficial for the economy of country as man power contributes a lot. The explanation behind quick spread of epidemic diseases in Pakistan incorporate packed urban areas, dangerous drinking water, deficient sanitation, poor financial conditions, low well-being mindfulness and insufficient immunization scope. Just about 60 million individuals in this nation do not have admittance to essential well-being offices. In addition, 90 million individuals have no fundamental sanitation. There is one specialist for 1,837 individuals, one dental practitioner for 46,498 people. One essential tends to 
14900 individuals and one clinic bed for 1503 people. This demonstrates a reasonable photo of our limitlessly denied well-being area. [14]

A substantial total of the financial backing is being spent on the therapeutic care and clinic cost in a situation where $33 \%$ of the populace, in Pakistan live beneath the destitution line and well-being offices remain broadly blocked off to the masses. Likewise, Pakistan has more than $98 \%$ out of the pocket well-being consumptions, where as more than $75 \%$ visits private Hospitals for human services. National general well-being consumption is $\$ 4$ per capita while add up to use on well-being is $\$ 18$ per capita. This mirrors the immense share of private medicinal services burning through (75.6\%). Social medical coverage covers just $5 \%$ of the populace yet speaks to $40 \%$ of the elected and common government spending on well-being [14]. We have studied that patients die due to late arrival of doctors, due to faulty operation techniques and rude behavior of administration and due to lack of cooperation of hospital management. 48 Millions Has been allocated for the health care system for the year 2017 including the National program on health by the Federal Government of Pakistan. As after $18^{\text {th }}$ amendment health is now provincial issue and the provincial governments allocated budgets accordingly.

There is no mature online system available that can provide ease to the people suffered from deadly diseases. There is no online helpline available that can guide people from rural and urban areas to find right place for their medical treatment. It is possible only when we would have available online feedback management system that can rate public medical facilities according to their performances and feedbacks from the patient visitors. Many hospitals are using web portals these days. However, these hospitals do not have such portal which can rate the doctors and administration of public hospitals via some feedback mechanism. For this purpose, in this article, we have proposed a system that will rate public hospitals, doctors, and the hospital management through feedback system. This system would run a reputation algorithm that would based on a trust management system. This system, in turn, would be able to generate true ratings of all the hospitals that are included in our system. These ratings help people in choosing right hospital for their deadly disease. This kind of feedback system has not been implemented yet in any medical institution within Pakistan.

The rest of the Paper is divided as follows: Section 2 describes the previous work on this research. Section 3 explains the methodology that is adapted in this research. Section 4 discussed the proposed solution that was developed during the research. Section 5 shows the results of our work. Section 6 concludes our work and future directions is given.

\section{Related Work}

Researchers [1] have measured Patient's expectations, perceptions and their experiences with health care system are the main determinants of patient's satisfaction. These domains are interconnected. Three categories of patient expectation include background expectation, interaction expectations, and action expectations. These expectations are affected by patient characteristics i.e. age, sex; ethnicity etc. Older people have lower expectations thus likely to be more satisfied with health care. Gender was found to be an inconsistent predictor of patient 
satisfaction, females tend to be lesser satisfied. People from the low social class were found to be more satisfied.

In the research [8] K-Nearest Neighbor algorithm was used which gives $1.7 \%$ better result than the other techniques used for this problem. Generally, Doctor Diagnosis patient through his tests, physical condition and patient's history, the amount of information may be insufficient, contain uncertainty, information may be misleading. For better results, they apply machine learning techniques for better classification and they applied this to Wisconsin Madison breast cancer problem.

In study [2] it was discussed that the health care system in Pakistan beset with numerous problems- structural fragmentation, gender insensitivity, resource scarcity and lack of inaccessibility and inefficiency. The goal of Health System (HS) reforms is to improve the technical and allocative efficiency of health care system, enhance the quality of services and make system more equitable. It is the dire need to enhance the system, in order to, bring quality health in country. Structural problems of health care system also require attention. Under-utilization, lack of quality, and scarcity of human, financial and technical resources are persistent problems. In short, Pakistan is in a need of a far- changing health sector reforms.

In [3] it was discussed about how to improve trust of user in long-term archiving. Electronic health records (EHRs) is a good example of long-term stored data. Attackers may temper the data without being noticed. For this purpose, it is needed to be addressed as a public key infrastructure, which can be trusted worldwide. However, this may not be practically possible. In this article, a long-term evaluation of Trust (LoT) is introduced which is reputation based, which alleviate the required trust assumptions. In this system, each document is signed periodically after some time. This is renewal procedure. This renewal depends upon date and time. If the document is signed through trusted time-stamp authority, it will be updated periodically. If a person wants to check whether its document is saved so, he/she will be retrieved with updated document.

In [4], [5], [6] and [10] the comparison of developed countries regarding health was carried out to show the reality that why Pakistan lack in healthcare system. China, Singapore, and USA improved a lot in healthcare system and facilitated their people. They have designed such systems to renovate the healthcare problems and letting the people know about diseases. These countries allocate a lot of budget of GDP to health. Bad governance, budget allocation, use of modern technology and usage of allocated budget properly is discussed in details.

In [7] the challenge of Private healthcare system is discussed in detail and it was found that the level and quality of care remains questionable in healthcare system. The result is low usage of public services when factors i.e. trust, perceived quantity, access, cost etc. are found weak. Pakistan has established a room for private sector to furnish and it is operating to play weakness in health care delivery to the poor people. Private sector has demonstrated great deal of responsiveness, hence winning the trust of clients. Pakistan is challenging the poor performance of health care system. Investments of government remained low. $1: 1099$ is doctor to patient ratio and $1: 1647$ is 
one bed to patient ratio in hospitals. The myth that the private health sector is an unimportant part in Pakistan has proven to be wrong. Private sector provides majority of the health care. It is undoubted fact that the major support to private sector is given by the government physicians practicing in the private arena.

In [8] it was discussed the comparisons of private and public hospitals. It was checked that public hospitals lack some fundamental facilities for the patients. Health sector of Pakistan is a mixed system of public, private, formal/non-formal and modern faith-based, all co-existing. The health indicators of Pakistan show a high population growth rate, gradually declining infant and maternal mortality rates. These indicators are not even good to be compared with neighboring countries. Budget allocated is also very low as per the requirements. By 2013, the number of hospitals are 1096, Basic Health Units 5527, Rural health centers 650, 5310 dispensaries, 167,759 doctors, 13,716 dentists, 86,183 nurses. There are 1099 patients per doctor and 13,411 patients for one dentist. On average 1647 persons per bed.

In [15], an authentic model has been proposed using cloud and sensor based network for trust and reputation based. In [16], they have proposed intelligent fuzzy logic system in network for reputation based model. In [17], different trust based reputation algorithm are used. In [18], using mobile sensor network an intelligent data gathering and efficient routing algorithm is used. In [19], for online reputation they introduced new framework that is based on fuzzy logic. In [20], sensing system introduced based on trust.

We need to make sure the quality and safety of services. Many private hospitals in developing countries have problems. But Private hospitals are not the ultimate solution to cater with health problems and also there is a grave problem of trust in Pakistan. There is no online system available that can gather feedback from community through automated way and disseminate information to the people. This information would help them finding right place for their medical care.

\section{Methodology}

It is identified in the previous section that there exist a problem of trust regarding medical treatment. For this purpose, we have developed a reputation-based feedback system, in order to, facilitate people. In the first phase, we have conducted a survey from people related to different communities in the form of questionnaire. However, results of survey were too much surprising. People used to prefer private hospitals over public hospitals even for major as well as for minor diseases. This is an alarming situation for country whose population is now more than 200 million.

We started our survey by incorporating google forms in our methodology. Google forms helps you to get people opinions in this busy world. So, we added four basic and comprehensive short questions for the survey and then we selected 500 most matured people around us and sent them the link of google forms. Based on their answers we have following .

In our first question, we asked people if they prefer private hospitals or public hospitals. More than $75 \%$ opted for private hospitals. The result is shown in Fig.01. 


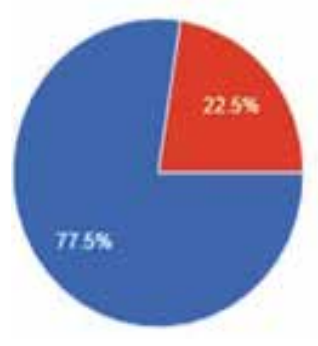

Fig.01. Feedback of $1^{\text {st }}$ Question

Therefore it is clear, that public hospitals do not have much capability to attract patients. This is due to lack of poor management and medical treatment.

In our second question, we had asked about the preference of public hospitals over private hospitals for the treatment of minor disease. The result has been shown in Fig.02. The result shows that more than $60 \%$ people prefer Private hospitals.
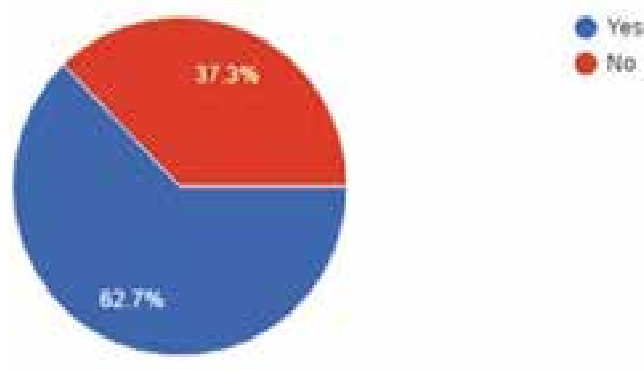

Fig.02. Feedback of $2^{\text {nd }}$ Question

However, the results are even more surprising when we had asked either to prefer public hospitals over private hospitals for deadly diseases. Approximately $70 \%$ of surveyors do not willing to go to the public hospitals for the treatment of deadly diseases like Cancer, Hepatitis etc. The result has been shown in Fig.03.
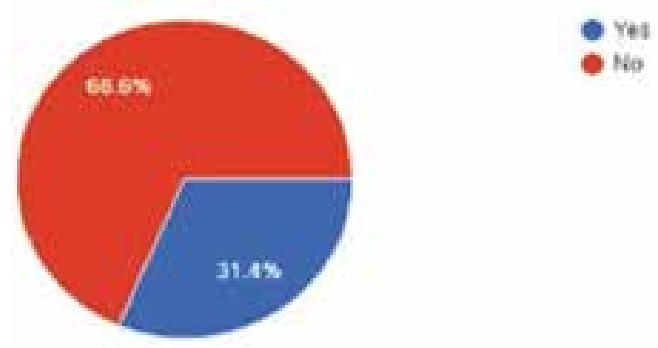

Fig.03. Feedback of $3^{\text {rd }}$ Question

The last question was to rate the current healthcare standard in Pakistan from Highest (5) to the Lowest (1).

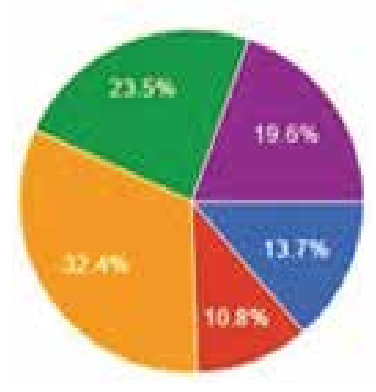

Fig.04. Feedback of $4^{\text {th }}$ Question

\section{Proposed Solution}

Keeping in view of the aforementioned feedbacks from our survey, we have developed a Reputation-based trust management system for improving public health care institutions in Pakistan. This is a web-based system that is user-friendly and can be accessible via our URL (www.rbhcs.net).

\subsection{How this system will work?}

This system carries a main feature of feedback. Feedback will enhance the capacity of system as it will get reviews of patients who are being checked up and are treated. Patients would be asked few questions when they visit a certain hospital for the treatment of deadly diseases 
like Ccancer, Hepatitis etc. In this system, the user will be given a form in which he/she has to give his/her particulars and answers to the questions asked in the form. Based on the results generated by these questions, an individual feedback is created. This feedback has a numerical value associated with it. Therefore, through this process, a unique feedback is generated which includes a particular hospital and a particular disease. The overall feedback from a number of patients helps in generating the hospital rating. Top rated hospital would be at the top of list for a particular disease. The system will be able to rate the diseases and will rate the doctors and hospital on the basis of their performance. Fig.04. shows our web-based reputation feedback system for health care.

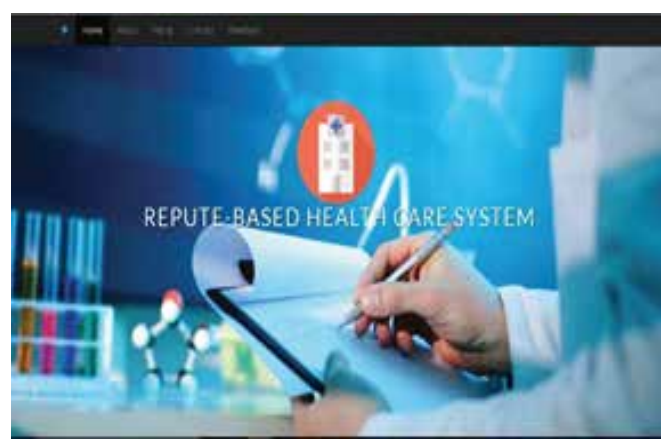

Fig.05 Our Proposed Reputation-based feedback system

Fig.05 shows following features which includes Rating and Feedback tab. A person after getting treatment from the Hospital will give feedback on his/her treatment. On the basis of cumulative feedback from a number of patients visiting a certain hospital, ratings would be generated.

\section{RESULTS}

If a person wants to get information of medical treatment for a particular disease, he or she will simply click on Disease tab and eventually get rating of hospital based on its performance. Fig.06 shows the Disease tab used by a person looking for treatment for a disease.
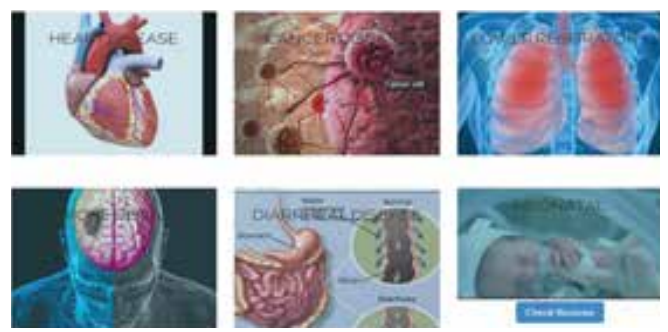

Fig.06 Disease Tab provides information about Hospitals

Let say, for example, a person is suffering from cancer and want to get information about well-reputed hospital treated cancer. Through clicking on the Disease tab, results of hospitals are shown on the basis of rating generated through feedback system. Fig.07 shows hospitals rating generated for Cancer disease treatment.

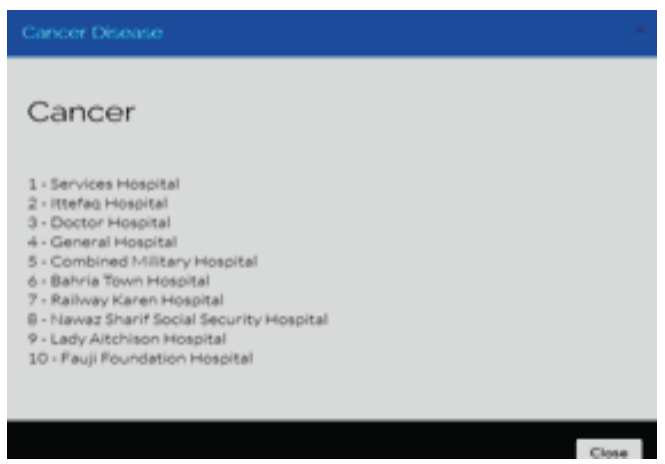

Fig.07. List of Hospitals according to ratings

The patient will be allowed to give his/her opinion about the doctors, staff and the hospital via this feedback form and rate the hospital and disease to tell the future patients whether they should come here or not? This 
will increase a level of competition amongst the hospital and they will try to convince the patient by providing him/her the best of facilities they can.

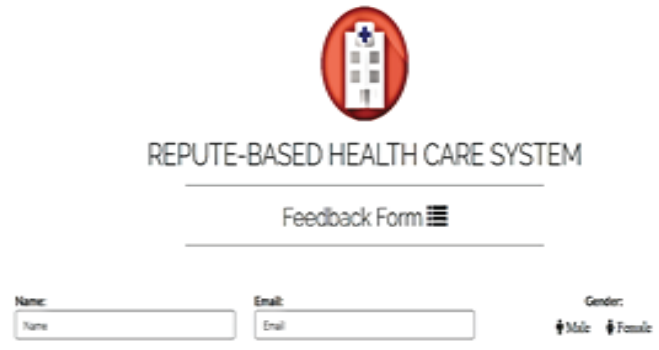

Fig 08. Web-based Feedback Form

In Fig.08, a web-based feedback form has been created for our system. The Patient visiting hospital must fill this feedback form. Several questions can be asked from the patient. Some questions are generic, others may be specific to the disease.

In this way, the system will give opportunity to the patients to give their precious opinions and will let them help administration to improve the healthcare system.

\section{Conclusion \& Future Work}

Pakistan is an emerging state in the world and with the passage of time population of Pakistan is also increasing. Increase in population also increases the demand of healthcare system. This increasing demand of healthcare facilities has become a question when no praise worthy facilities is given to the people by the government which is responsible for it. In our research, we have developed an online health care system. This system actually works on reputation of hospitals that comes after the feedback from the community resulting in enhancement of trust of people.

In the future, we will work on development of
Android-based application that would help people finding right medical care center for their treatment. We will also improve our existing system by adding some new features like online doctor help, Doctor-Patient trust etc. Feedback system is used to maintain a record of hospitals, diseases and the administration to improve the standard of medical treatment when it comes to the phase of competition. There is a dire need to put proper check and balance in our healthcare system if at all we want to improve it. Feedback system will benefit us in doing so. This research will help a lot in bringing improvement in the health sector of Pakistan.

\section{References}

[1] Nasser, M. Zahidie, A., Shaikh, B.T. (2012), Determinants of patient's satisfaction with healthcare system in Pakistan: a critical overview. Pakistan journal of public health, 2(2), 52-61.

[2] A. Islam, (2014), Health Sector reforms, why is it needed? JPMA 52-3(2002) 95:100.

[3] M. Vigil, D.D., Shaikh M.H., Sascha H., J. Buchamamn, Max M. (2016), A reputation based trust system for long-term achieving. IARIA, 2016, ISBN: 978-1-61208-493-0.

[4] S.T. Meghani \& N.S. Punjani, (2014), Comparison and analysis of healthcare system: Pakistan Versus China, 2014.

[5] Laila A. Amir A., Rehana A., (2014), Healthcare system, Pakistan Versus Iran, International journal of innovative research and development, Volume 3 
issue 7, July 2014.

[6] Hina Karim (2017), Analysis of Health Care Delivery System in Pakistan and Singapore, IJNES 2017.

[7] Babur Tasneem Shaikh (2015), Private Sector in healthcare delivery: A reality and challenge in Pakistan, 27(2), 2015.

[8] Ather H. Akbar, Wimal R., Adiqa K (2009), Demand for public health care in Pakistan, 48:2 (Summer 2009) pp 144-153.

[9] Neelam S.P. S. Shams \& S.M. Bhanji (2014), Analysis of healthcare delivery system: Pakistan Versus United States, International Journal of Endorsing Health Science Research, Volume 2 Issue 1. 2014.

[10] Piero B., Clandiu D., Daniel O. \& Shahmehri. An integration reputation based and policy based trust management, IST -506779 .

[11] A. Yadav, S. Chakravarty \& Ritu S. (2015), A Survey of implicit trust on social networks. IEEE 2015.

[12] Uzma A. \& Anam Y. (2013) State of health in Pakistan: An overview. 18: SE, PP. 233-247(2013).

[13] Shariq K., Richard S., Salman G, e-Health readiness assessment: Promoting 'hope' in the healthcare institutions of Pakistan, Vol 44 No. 1

[14] M. Akram, F. Jehangir Khan (2007), "Health care services and government service in Pakistan", Pakistan Institute of Development Economics (PIDE).

[15] Chunsheng Z. An authenticated trust and reputation calculation and management system for cloud and sensor networks integration. IEEE Trans Inform Forens Secur 2015; 10: 118-131.

[16] Mary Subaja Christo1*, Meenakshi S2 and Subhashini R3An intelligent fuzzy beta reputation model for securing information in $\mathrm{P} 2 \mathrm{P}$ health care applications, Biomedical Research (2017) Volume 0, Issue 0

[17] Riccardo P. S-Kademlia: A trust and reputation method to mitigate a Sybil attack in Kademlia. Comp Netw 2016; 94: 205-218.

[18] Jothimuneeswari S, Ganapathy S, Kannan A. Intelligent data gathering and energy efficient routing algorithm for mobile wireless sensor networks. As J Inform Technol 2016; 15: 921-927.

[19] Edy P, Andreas M, Philippe CM, Witold P. FORA-A fuzzy set based framework for online reputation management. Fuzzy Sets Sys 2015; 269: 90-114.

[20] Hayam M, Sonia BM, Omar H, Osama Y, Mohiy H, Lionel B. Trust management and reputation systems in mobile participatory sensing applications: A survey. Comp Netw 2015; 90: 49-73.

[21] www.rbhes.net 